Esta publicación cientifica en formato digital es continuidad de la revista impresa ISSN-Versión Impresa 0798-1406 / ISSN-Versión on line 2542-3185Depósito legal pp
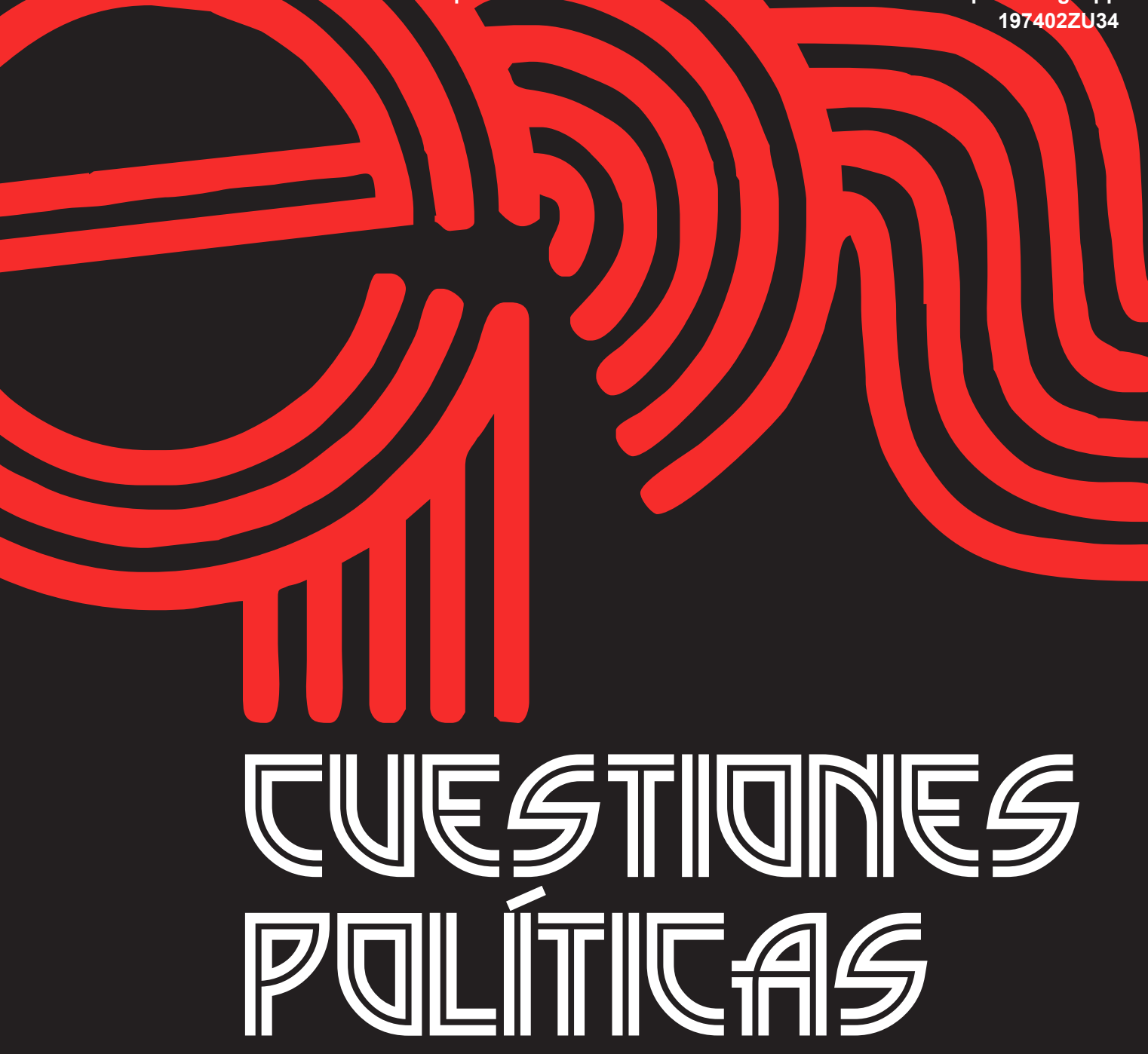

Instituto de Estudios Políticos y Derecho Público "Dr. Humberto J. La Roche" de la Facultad de Ciencias Jurídicas y Políticas de la Universidad del Zulia Maracaibo, Venezuela
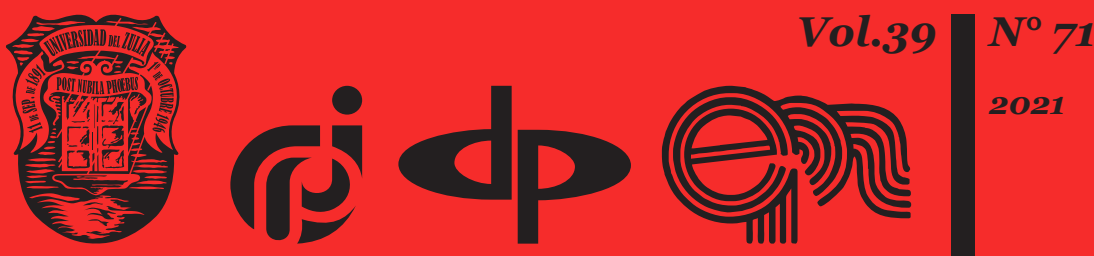


\title{
Significados políticos y jurídicos del uso global de las criptomonedas
}

\author{
DOI: https://doi.org/10.46398/cuestpol.3971.00 \\ Jorge J. Villasmil Espinoza *

\section{Resumen}

El uso global de los criptoactivos es una práctica generalizada en el año 2021 entre personas y empresas que están interesadas en participar en nuevas formas de negocios que, pudieran ser muy lucrativos en el mediano plazo, además de representar una nueva concepción monetaria de carácter digital que no está controlada por ningún banco central, ni emitida por ningún estado. El propósito de este breve ensayo es presentar el volumen 39, número 71 de Cuestiones Políticas, mediante el desarrollo de un conjunto de reflexiones sobre el alcance y significado de las criptomonedas en el mundo de hoy. Se concluye que, la sola idea de acuñar una moneda en formato digital del sujeto ficticio Satoshi Nakamoto creador del Bitcoins, resulta vanguardista para todas las personas que quieren realizar intercambios de valores sin ninguna mediación centralizada de instituciones financieras privadas o públicas, mediante una red anomia de acceso abierto conformada por sujetos libres e iguales, que puede dar al traste con el control monetario de las economías por parte de los gobiernos, al tiempo que crea alternativas para las monedas Fiat. Entre los aspectos negativos destacan el uso creciente de enérgica eléctrica que requieren las criptos para poder desarrollar sus operaciones.

Palabras clave: criptomonedas; criptoactivos; anarquismo digital; economía global; nueva política económica. 


\title{
Political and legal meanings of the global use of cryptocurrencies
}

\begin{abstract}
The global use of crypto assets is a widespread practice in 2021 among people and companies that are interested in participating in new forms of business that could be very lucrative in the medium term, in addition to representing a new monetary conception of a digital nature that is not controlled by any central bank, nor issued by any state. The purpose of this short essay is to present volume 39, number 71 of Political Issues, by developing a set of reflections on the scope and meaning of cryptocurrencies in today's world. It is concluded that, the mere idea of minting a currency in digital format of the fictitious subject Satoshi Nakamoto creator of Bitcoins, is avant-garde for all people who want to carry out exchanges of values without any centralized mediation of private or public financial institutions, through an open access anomie network made up of free and equal subjects, that can death rate the monetary control of economies by governments, while creating alternatives for Fiat currencies. Among the negative aspects are the growing use of energetic electricity that cryptos require to be able to develop their operations.
\end{abstract}

Keywords: cryptocurrencies; crypto assets; digital anarchism; global economy; new economic policy.

Finalizando el 2021 el uso de las criptomonedas es un fenómeno global entre personas y empresas que están interesadas en participar en nuevas formas de negocios, sin el control restrictivo de los gobiernos que normalmente hacen un uso político e ideológico de las economías en funcionar de beneficiar particulares intereses en detrimento de otro. Desde la emergencia del Bitcoins en 2008, han surgido cientos o quizás miles de criptoactivos con diferentes usos y valores, pero en líneas generales:

Las criptomonedas son monedas digitales que se usan para el intercambio de bienes o servicios. Nacieron a partir de la crisis financiera en el año 2008, y se comenzaron a usar en transacciones por Internet.

No obstante, en la actualidad no solo las empresas electrónicas las están aceptando como formas de pago, sino que algunas compañías de renombre también se sumaron debido a los beneficios que brindan (Telesur.net, 2021: s/p).

Este creciente interés por las criptomonedas también conocidas como criptoactivos es el que permite indagar científicamente desde los dominios de la ciencia política, cual es el significado político y jurídico de estas. Obviamente es difícil tratar de reflexionar sobre estas cuestiones en abstracto, esto es, sin analizar lo sucedido en ciertos contextos nacionales 
específicos, pero, de cualquier modo, es posible efectuar algunas presiones al respecto con algún alcance internacional.

Conviene recordar que la capacidad para producir monedas validas como unidades de cambio en los mercados más allá del trueque, es, desde la antigüedad, un signo distintivo del poder del estado para intervenir en las realidades económicas de las sociedades sometidas a su autoridad. Incluso si se piensa en el estado como una estructura ficcional al servicio de ciertos sectores de poder, habría que reconocer de igual modo que el fenómeno monetario es un dispositivo de poder y control social de las diversas formas de gobierno históricamente existente.

Este este orden de ideas, más allá de ciertas experiencias particulares con algún alcance local o regional, el monopolio de producción de las monedas y la política monetaria de los estados en general, nunca había sido tan amenazada desde el surgimiento del Bitcoins, moneda digital que demuestra que en la práctica es posible estructurar mancomunadamente dinero legítimo con alcance global y alto valor en el mercado --de hecho para el momento que se escriben estas líneas, noviembre de 2021 su costo esta próximo a los 60.000 dólares americanos--, sin la regulación del estado, ni la intermediación de ninguna institución financiera publica o privada.

En consecuencia, el todo indica que el principal significado político de este fenómeno esta vinculado a la creación de espacios virtuales intersubjetivos menos regulados y mas libres que permiten a las personas ahorra e intercambiar bienes y servicios de forma autónoma, ante lo cual hay al menos dos posibles alternativas por parte de los gobiernos: por un lado, tratar de regular todos lo concerniente al uso, distribución e intercambio de los criptoactivos cosa sumamente difícil y que contraviene la filosofía anarco-libertaria de la mayoría de las criptos o, incluso, impedir las transacciones con las mismas como parece ser el caso de India, China y Rusia o; por el contrario, auspiciar su producción y reproducción en la economía como han hecho países como el Salvador o Brasil, con un resultado aún incierto en términos de bienestar social o incremento de la riqueza colectiva.

En los dominios jurídicos, todo indica que la mayoría de los estados nacionales tienen que adecuar sus cuerpos normativos al impacto de este fenómeno relativamente reciente, ante lo cual hay también al menos dos opciones claras: primero, avalar legalmente el uso y disfrute de las criptomonedas, aunque con algunas reservas y regulaciones en función del verdadero interés de la ciudadanía o; por el contrario, segundo, plegarse acríticamente a la agenda legislativa internacional que diseñen los estados y corporaciones hegemónicas para seguir perpetuando el dominio de ciertas divisas como el dólar o el euro en las economías de los países periféricos. 
¿Cuál será el futuro de las criptomonedas? ¿se trata de un fenómeno irreversible que marca un antes y un después en la historia económica de la humanidad? Muy seguramente en las próximas décadas tendremos respuestas claras para este tipo de preguntas, no obstante, la sola idea de acuñar una moneda en formato digital del sujeto ficticio Satoshi Nakamoto creador del Bitcoins, resulta vanguardista para todas las personas que quieren realizar intercambios devalores sin ninguna mediación centralizada, mediante una red anomia de acceso abierto conformada por sujetos libres e iguales, que, llegado el caso, puede dar al traste con el control monetario de las economías por parte de los gobiernos, al tiempo que crea alternativas para el uso de las monedas Fiat. Sin embrago, no todo es bueno con la criptos, entre los aspectos negativos destacan el uso creciente de enérgica eléctrica que requieren las granjas de minería digital para garantizar la cadena de bloques que hacen posible las tracciones cotidianas con estos instrumentos de pago.

\section{Referencias Bibliográficas}

TELESUR.NET. 2021. “Criptomonedas en la economía mundial” En: Telesur en profundidad. Disponible en línea. En: https://www.telesurtv.net/ telesuragenda/criptomonedas-economia-mundial-20210908-0026. html. Fecha de consulta: 12/10/2021. 

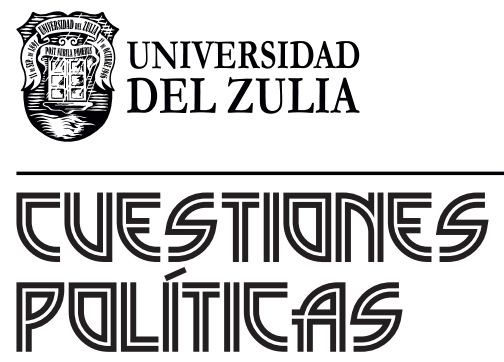

Vol. 39 N $^{\circ} 71$

Esta revista fue editada en formato digital y publicada en diciembre de 2021, por el Fondo Editorial Serbiluz, Universidad del Zulia. Maracaibo-Venezuela 